\title{
Thermodynamics of galaxy clusters in modified Newtonian potential
}

\author{
Abdul W. Khanday, $1, *$ Sudhaker Upadhyay, $2,3,4,5,+$ and Prince A. Ganai ${ }^{6, t}$ \\ ${ }^{1}$ Department of Physics, National Institute of Technology Srinagar, \\ Jammu and Kashmir-190006, India. \\ ${ }^{2}$ Department of Physics, K. L. S. College, Nawada, Bihar 805110, India \\ ${ }^{3}$ Department of Physics, Magadh University, Bodh Gaya, Bihar 824234, India \\ ${ }^{4}$ Inter-University Centre for Astronomy and Astrophysics (IUCAA) Pune, Maharashtra-411007 \\ ${ }^{5}$ School of Physics, Damghan University, Damghan, 3671641167, Iran \\ ${ }^{6}$ Department of Physics, National Institute of Technology \\ Srinagar, Jammu and Kashmir-190006, India.
}

\begin{abstract}
We study the thermodynamics of galaxy clusters in a modified Newtonian potential motivated by a general solution to Newton's "sphere-point" equivalence theorem. We obtain the $N$ particle partition function by evaluating the configurational integral while accounting for the extended nature of galaxies (via the inclusion of the softening parameter $\epsilon$ into the potential energy function). This softening parameter takes care of the galaxy-halos whose effect on structuring the shape of the galactic disc has been found recently. The spatial distribution of the particles (galaxies) is also studied in this framework. A comparison of the new clustering parameter $b_{+}$to the original clustering parameters is presented in order to visualize the effect of the modified gravity. We also discuss the possibility of system symmetry breaking via the behavior of the specific heat as a function of temperature.
\end{abstract}

\footnotetext{
*Electronic address: abdulwakeelkhanday@gmail.com

${ }^{\dagger}$ Electronic address: sudhakerupadhyay@gmail.com

${ }^{\ddagger}$ Electronic address: princeganai@nitsri.net
} 


\section{INTRODUCTION}

Like stars and galaxies, galaxy clusters are one of the fundamental building blocks of the universe. These systems form the topmost level of the hierarchical structure formation and possess the most extreme masses and dimensions of all celestial objects. Due to the complex optical appearance, the systematic study of these systems hasonly recently begun. Advanced observational techniques have helped a lot in their proper understanding. Optical and X-ray observations have led to a deep understanding of the evolution and composition of these larger systems. The famous Sunyaev-Zeldovich (SZ) effect and the gravitational lensing effect have added important details to our knowledge on these systems. Observations and theoretical studies have led to the emergence of $\Lambda$ cold dark matter $(\Lambda \mathrm{CDM})$ model as a standard cosmological model. This model is based on a spatially flat universe, a cosmological constant responsible for the accelerated expansion at late times and a structure seeded by quantum fluctuations at very early times during the period of inflation.

"Messier" was the first to list 103 nebulae in his catalog, 30 of which are now identified as galaxies. Herschel was a pioneer in the study of large scale structure of universe. Herschel classified around 2500 nebulae in his lifetime and recognized several groups of galaxies, such as Ursa Major, Leo, Hydra and NGC 4169 etc. In the mid 1920s Hubble proved that the nebulae were extra-galactic in nature and bona fide galaxies like Milky way [1]. In 1927 Lundmark started his investigation of the formation mechanism of galaxy clusters for the first time. Zwicky [2] pioneering work on estimating the mass of galaxy clusters and establishing the need for extramatter, now known as dark matter (DM). pioneered the work of the estimation of mass of galaxy clusters and established the need for extra-matter now known as dark matter (DM).

The theoretical models of cluster formation employ techniques focusing on any particular aspect of clustering that they aim to understand. The initial conditions, DM mass budget and gravity determine many important bulk properties. The self-similar model by Kaiser is one such simple model [3]. This model predicts cluster properties close to observational findings. The closed box nature of cluster potential provides ideal laboratory conditions to understand galaxy formation and inside processes.

The models that describe the modifications to the standard general relativity (GR) gravity 
have implications for cosmic evolution as well as that of density perturbations and, therefore, for cluster formation. For example, $f(R)$ models which modify gravity by the inclusion of a general function $f(R)$ of the Ricci curvature scalar in the Einstein-Hilbert action enhance the gravitational forces on scales appropriate for structure formation [4].

The changes to the gravity law have prompted the study of the statistical and thermodynamic properties of galaxy clusters in light of the modified potentials [5 9$]$. For instance, the effect of quantum correction on the thermodynamics of galaxy clusters were studied in [10] and the effect of power-law modification to Newtonian potential on the spatial distribution function have been studied in Ref. [11]. Similarly, the effect of the expansion of the universe on the clustering of galaxies has been discussed in Ref. [12] and the effect of dynamical dark energy on the galactic clustering has also been studied [13]. Also, the expansion effect of the universe on the galaxy clustering has been investigated in Ref. [14]. Within these studies, it has been observed that the modified gravity law affects the thermodynamic and statistical properties of galaxy clusters considerably.

In the roots of the Newton's gravity the theorem of "sphere-point" equivalence lies. This principle helped Newton to relate the terrestrial gravity (apple falling towards earth) to the orbital motion of planets. We have realized Newtonian gravity as the weak-field limit of GR which enables communication between observations and prediction. It has been shown in Ref. [15] that the force function satisfying Newton theorem is given by

$$
F(r)=A r^{-2}+B r
$$

where $A$ and $B$ are constants related to the gravitational constant $G$ and the cosmological constant $\Lambda$ (within numerical factors), respectively. The force parameter $B$ intended to be such that the additional force is attractive. The weak-field GR thus ensures that any matter, seen or unseen [44], at large galactic scales interacts by the law of Eq. (1).

Any matter (seen or unseen) at large galactic scales interacts via the equation (11) [16]. Equation (11) defines a non-force-free field inside the spherical shell, thus contradicting the Newtonian concept of a force-free field inside. However, the non-force-free field inside the shell agrees well with the observation of the effect of the galactic halos on the structure of galactic disks [17]. The second term in the equation (11) corresponds to the cosmological constant term in the Newtonian 
formulation of the Friedmann cosmological equation [15]. Although there have been numerous studies on galaxy clustering under modified gravity, the most important approach of modified weak-field GR has been overlooked. We try to bridge this gap.

In this article, we quantitatively study the effect of the extra term ( $r$ dependency) present in Newton's law on the statistical distribution of galaxies. We also study the effects of this force form on the thermodynamic equations of state, viz. free energy, entropy, and chemical potential, etc. The effect of this correction on various thermodynamic quantities and the general distribution function is also observed. We also study the behavior of specific heat as an indicator of phase transition. The correction term shows up in the form of a modified clustering parameter which estimates the extent of correlation among different system particles.

\section{PARTITION FUNCTION OF THE GRAVITATING BODIES UNDER MODIFIED POTENTIAL}

The force function given in (11) corresponds to a monogenic and conservative system and related to the potential via the relation $F=-\frac{\partial \Phi}{\partial r}$. Utilizing the standard integral, $\Phi=\int-F d R$, the potential energy function $\Phi$ takes the form [18]

$$
\Phi(r)=-\frac{G M^{2}}{r}-B r^{2},
$$

where $B=M \Lambda c^{2} / 6$ (for numerical value see e.g. [18]). The partition function for $N$-body system of point particles interacting gravitationally and each having mass $M$ and momenta $p_{i}$ is given by [19]

$$
Z_{N}(T, V)=\frac{1}{\lambda^{3 N} N !} \int d^{3 N} p d^{3 N} r \exp \left(-\left[\sum_{i=1}^{N} \frac{p_{i}^{2}}{2 M}+\Phi\left(r_{1}, r_{2}, \ldots r_{N}\right)\right] T^{-1}\right),
$$

where $\lambda$ is a normalization factor.

Performing the integration over momentum space, we have

$$
Z_{N}(T, V)=\frac{1}{N !}\left(\frac{2 \pi M T}{\Lambda^{2}}\right) I_{N}(T, V)
$$


where $I_{N}(T, V)$ is configuration part of integral (3) and given by

$$
\begin{aligned}
I_{N}(T, V) & =\int \ldots . \int \prod_{1 \leq i<j \leq N} \exp \left(\left[-\Phi\left(r_{i}-r_{j}\right) T^{-1}\right]\right) d^{3 N} r \\
& =\int \ldots . \int \prod_{1 \leq i<j \leq N} \exp \left(\left[-\Phi\left(r_{i j}\right) T^{-1}\right]\right) d^{3 N} r .
\end{aligned}
$$

The gravitational potential energy depends on the relative positions only, $r_{i j}=r_{i}-r_{j}$, and, in general, estimated as the sum of potential energy of all pairs, i.e.

$$
\Phi\left(r_{1}, r_{2}, \ldots, r_{N}\right)=\sum_{1 \leq i<j \leq N} \Phi\left(r_{i j}\right)
$$

The configuration integral can be solved by introducing a two-point function, $f_{i j}[19]$. Begin equation

$$
f_{i j}=\exp \left(-\frac{\Phi_{i j}}{T}\right)-1
$$

to define the two-point function. The function $f_{i j}$ vanishes in the absence of interactions. The configuration integral takes the following form:

$$
I_{N}(T, V)=\int \ldots \int\left(1+f_{12}\right)\left(1+f_{13}\right) \ldots\left(1+f_{N-1, N}\right) d^{3} r_{1} d^{3} r_{2} \ldots d^{3} r_{N}
$$

For point masses, the Hamiltonian function diverges at $r_{i j}=0$. This discrepancy is eliminated by introducing a parameter $\epsilon$, which also accounts for the extended nature of galaxies, where $0.01 \leq \epsilon \leq 0.05$ is measured in cell size units. [20]. The interaction potential energy between galaxies takes on the form after the softening parameter $\epsilon$ is introduced

$$
\Phi=\left[\frac{-G M^{2}}{\left(r_{i j}^{2}+\epsilon^{2}\right)^{1 / 2}}-B r_{i j}^{2}\right] .
$$

Because the second term does not diverge as $r_{i j} \rightarrow 0$, the softening parameter $\epsilon$ is not introduced. The configuration integral for $N=1, I_{1}(T, V)$, takes the value

$$
I_{1}(T, V)=1
$$

Begin equation

$$
I_{2}(T, V)=4 \pi V \int_{0}^{R}\left[1+\frac{1}{\left(r^{2}+\epsilon^{2}\right)^{(1 / 2)} T}+B r^{2}\right] r^{2} d r
$$


to calculate the configuration integral for $N=2$. Here we have used the fact that the effect of the long-range mean gravitational field is exactly balanced by the expansion of the universe [21]. Upon solving, the above integral yields

$$
I_{2}(T, V)=V^{2}\left[1+\frac{3}{2} \frac{G M^{2}}{R T}\left(\sqrt{1+\frac{\epsilon^{2}}{R^{2}}}+\frac{\epsilon^{2}}{R^{2}} \log \frac{\frac{\epsilon}{R}}{1+\sqrt{1+\frac{\epsilon^{2}}{R^{2}}}}+\frac{2 B}{5 G M^{2}} R^{3}\right)\right] .
$$

In a more compact form, this equation can be written as

$$
I_{2}(T, V)=V^{2}\left[1+\frac{3}{2} \frac{G M^{2}}{R T}\left(C_{1}+C_{2}\right)\right]
$$

where

$$
\begin{aligned}
C_{1} & =\sqrt{1+\frac{\epsilon^{2}}{R^{2}}}+\frac{\epsilon^{2}}{R^{2}} \log \frac{\epsilon / R}{1+\sqrt{1+\frac{\epsilon^{2}}{R^{2}}}}, \\
C_{2} & =\frac{2 B R^{3}}{5 G M^{2}} .
\end{aligned}
$$

In general, the value of the configurational integral for higher values of $N$ is given by

$$
I_{N}(T, V)=V^{N}\left[1+\left(C_{1}+C_{2}\right) \eta\right]^{N-1},
$$

where

$$
\eta=\frac{3}{2} \frac{G M^{2}}{R T}
$$

Using equations (44) and (14), the grand partition function for the system of $N$ gravitationally interacting galaxies is given by

$$
Z_{N}(T, V)=\frac{1}{N !}\left(\frac{2 \pi M T}{\lambda^{2}}\right)^{3 N / 2} V^{N}\left[1+\left(C_{1}+C_{2}\right) \eta\right]^{N-1}
$$

This partition function includes the corrected parameter $C_{2}$ together with the uncorrected parameter $C_{1}$.

\section{THERMODYNAMICS OF THE SYSTEM OF GALAXIES}

Once the partition function is known, we can derive the thermodynamic equations of state for the system. The Helmholtz free energy carries all the useful information about the system that the partition function carries, and these are connected by the relation $F(V, N, T)=$ 
$-T \ln Z_{N}(T, V)$. For the system of galaxies, $F$ can be written as

$$
F(V, N, T)=-T \ln \left[\frac{1}{N !}\left(\frac{2 \pi M T}{\lambda^{2}}\right)^{3 N / 2} V^{N}\left[1+\left(C_{1}+C_{2}\right) \eta\right]^{N-1}\right] .
$$

We approximate $N-1 \approx N$ for the system of galaxies with a large $N$ value. With this approximation, equation (17) can be simplified to

$$
F=N T\left(\ln \frac{N}{V} T^{-3 / 2}\right)-N T-N T \ln \left[1+\left(C_{1}+C_{2}\right) \eta\right]-\frac{3}{2} N T \ln \left(\frac{2 \pi M}{\lambda^{2}}\right) .
$$

Here, we observe that within the weak-field limit of GR the Helmholtz free energy of the system is modified by term having parameter $C_{2}$ in the logarithmic term.

The entropy of the system of galaxies is computed for the above partition function using the following relation:

$$
S=T\left(\frac{\partial \log Z}{\partial T}\right)_{N, V}+\log Z
$$

Substituting the values from equation (16) into equation (19), the entropy of the system takes the following functional form:

$$
\begin{aligned}
S & =N \ln \left(\frac{V}{N} T^{3 / 2}\right)+N \ln \left[1+\left(C_{1}+C_{2}\right) \eta\right]-3 N \frac{\left(C_{1}+C_{2}\right) \eta}{1+\left(C_{1}+C_{2}\right) \eta} \\
& +\frac{5}{2} N+\frac{3}{2} N \ln \frac{2 \pi M}{\lambda^{2}} .
\end{aligned}
$$

This relationship can be further simplified to

$$
\frac{S}{N}=\ln \left(\frac{V}{N} T^{3 / 2}\right)-\ln \left[1-b_{+}\right]-3 b_{+}+\frac{5}{2}+\frac{3}{2} \ln \frac{2 \pi M}{\lambda^{2}},
$$

where $b_{+}=\frac{\left(C_{1}+C_{2}\right) \eta}{1+\left(C_{1}+C_{2}\right) \eta}$ is a clustering parameter that takes values between 0 and 1 and estimates the strength of interaction. The Newtonian clustering parameter $b_{e}$ psilon defined in [19] is related to the clustering parameter $b_{+}$via the relationship

$$
b_{+}=\frac{b_{\epsilon}\left(1-C_{2} \eta\right)+C_{2} \eta}{1+C_{2} \eta-b_{\epsilon} C_{2} \eta}
$$

where $b_{\epsilon}=\frac{C_{1} \eta}{1+C_{1} \eta}$. In the above relation, when $C_{2} \rightarrow 0, b_{+} \rightarrow b_{\epsilon}$.

The variation of the clustering parameter $b_{+}$with the strength of the correction term can be visualized from the Fig. 1. The clustering becomes stronger as the value of the correction term increases. 


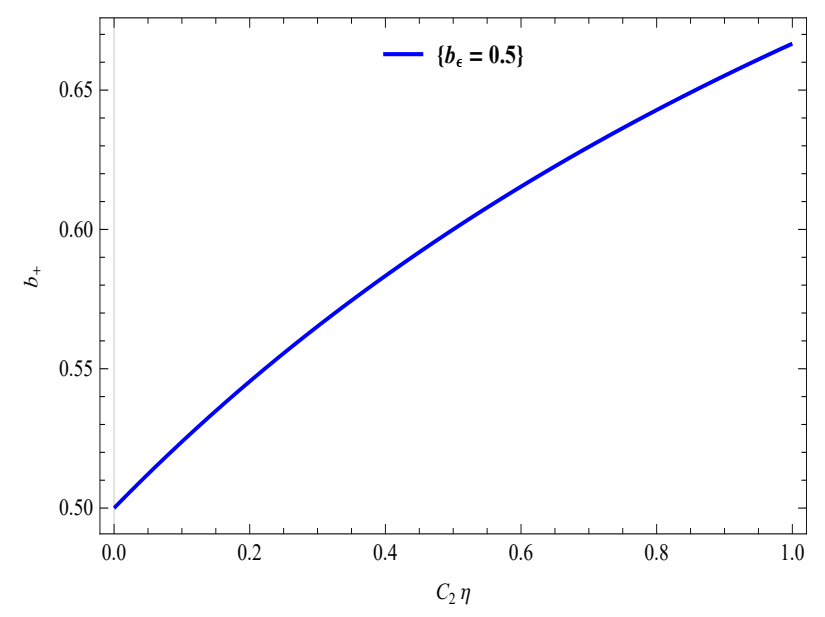

FIG. 1: For a fixed value of the unmodified clustering parameter $b_{\epsilon}$, the figure depicts the variation of the modified clustering parameter $b_{+}$with the strength of the correction term $C_{2} \eta$.

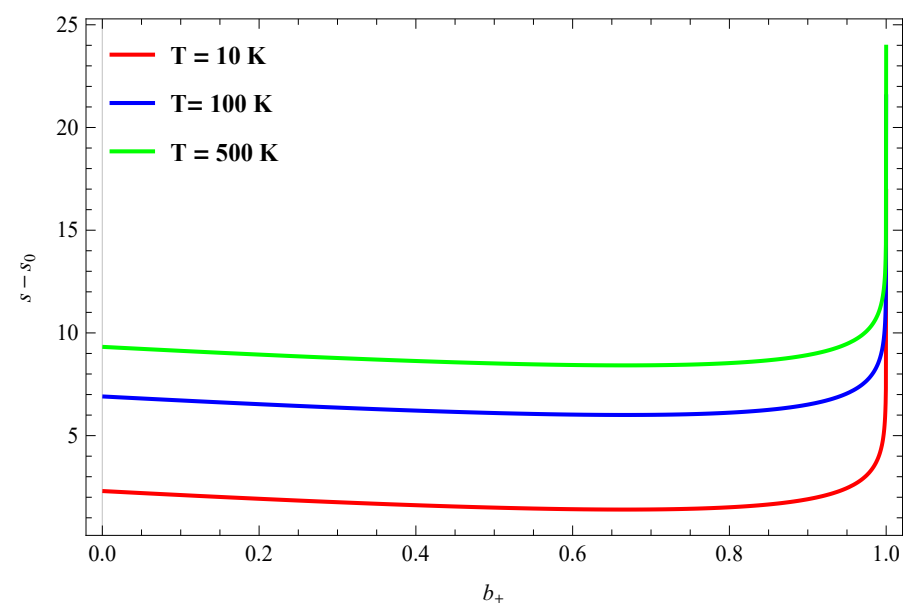

FIG. 2: The graph depicts the isothermal variation of specific entropy $\left(S-S_{0}\right)$ as a function of clustering parameter $b_{+}$for three different system temperatures and a fixed average density (barn).

If $\bar{n} T^{-3 / 2}$ is constant, the gravitational contribution to entropy $S-S_{0}=-\log \left[1-b_{+}\right]-3 b_{+}$ reduces total entropy significantly for small $b_{+}$but makes it infinite as $b \rightarrow 1$ due to a logarithmic divergence. This is evidence of the undergoing phase-transition of the system as it becomes more and more clustered. For $b=0$, the entropy reduces to that of a non-interacting classical gas.

Utilizing free energy (18) and entropy (20), the internal energy for the system of galaxies can 
be derived using the relation, $U=F+T S$, as

$$
U=\frac{3}{2} N T\left[1-2 b_{+}\right]
$$

The pressure is related to Helmholtz free energy via the relation $P=-\left(\frac{\partial F}{\partial V}\right)_{N, T}$, which involves the first derivative of free energy w.r.t volume $V$, keeping particle number, $N$, and temperature, $T$, fixed. The pressure of the system of galaxies reads:

$$
P=\frac{N T}{V}\left[1-b_{+}\right]
$$

The chemical potential, $\mu$, measures the change in the internal energy of the system if a particle is added to the system while keeping volume and temperature constant, i.e., $\mu=\left(\frac{\partial F}{\partial N}\right)$. Using the relation for Helmholtz free energy, the chemical potential takes the following functional form:

$$
\mu=T\left(\ln \frac{N}{V} T^{-3 / 2}\right)+T \ln \left[1-b_{+}\right]-T b_{+}-\frac{3}{2} T \ln \left(\frac{2 \pi M}{\lambda^{2}}\right) .
$$

The above chemical potential can further be expressed as

$$
\frac{\mu}{T}-\mu_{0}=\left(\ln \frac{N}{V} T^{-3 / 2}\right)+\ln \left[1-b_{+}\right]-b_{+} .
$$

The behavior of the chemical potential as a function of order parameter $b_{+}$is shown in the following graph (Fig. 3).

\section{GENERAL DISTRIBUTION FUNCTION}

Now, we use the results derived above to obtain the functional form of the gravitational quasiequilibrium distribution function (GQED), $F(N)$, for the cosmological many-body problem. We treat our system as an ensemble of sub-regions all of the same volume. We let the number of galaxies and their mutual gravitational energy fluctuate among the sub-systems. Thus, our system is a grand-canonical system characterized by a chemical potential $\mu$ and a temperature $T$. The partition function (a weighted sum of all canonical partition functions) for the grand canonical ensemble is defined by

$$
Z_{G}(T, V, z)=\sum_{N=0}^{\infty} e^{\frac{N \mu}{T}} Z_{N}(T, V),
$$




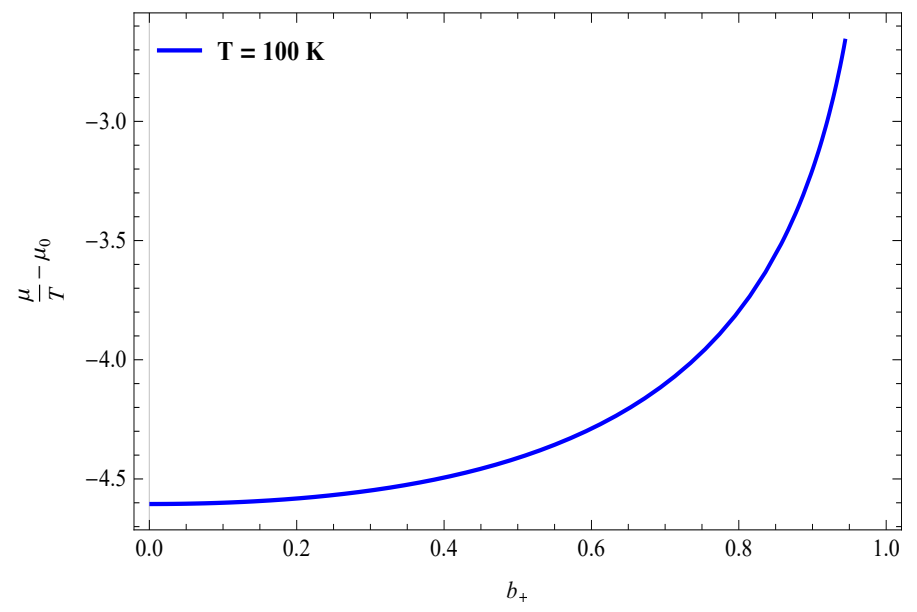

FIG. 3: For a fixed value of $\bar{n} T^{3 / 2}$, the figure depicts the variation of chemical potential $\mu / T-\mu_{0}$ as a function of clustering parameter $b_{+}$. The chemical potential is negative as the addition of a single particle decreases the total gravitational binding energy of the system.

where $z$ is the system activity given by $z=\exp \left(\frac{\mu}{T}\right)$.

The partition function for the system of galaxies interacting through the modified Newtonian potential is given by

$$
\log Z_{G}=\frac{P V}{T}=N\left(1-b_{+}\right)
$$

The probability of finding $N$ particles in volume $V$ is calculated via the relation

$$
F(N)=\frac{\sum_{i} e^{\frac{N \mu}{T}} e^{\frac{U_{i}}{T}}}{Z_{G}(T, V, z)}=\frac{e^{\frac{N \mu}{T}} Z_{N}(T, V)}{Z_{G}(T, V, z)} .
$$

Using equations (16), (27), and (29), the distribution function for the system of galaxies is given as

$$
F(N)=\frac{\bar{N}}{N !}\left(1-b_{+}\right)\left[\bar{N}\left(1-b_{+}\right)+N b_{+}\right]^{N-1} e^{-N b_{+}-\bar{N}\left(1-b_{+}\right)} .
$$

According to Ref. [22], this thermodynamic distribution function agrees well with the Zwicky catalogue distribution of galaxies, and the clustering parameter is $b_{+} \approx 0.75$. A more recent cluster catalogue described in [23] based on the Sloan Digital Sky Survey 3, and containing 132, 684 galaxy clusters in the $0.05 \leq z \leq 0.8$ redshift range, also satisfies the distribution function described in equation (30). The distribution function $F(N)$ has a Poisson component modified 
by the corrected clustering parameter $b_{+}$. The pure Poisson distribution is restored in the limit $b_{+} \rightarrow 0$. Remarkably, the basic structure of the distribution function is similar to that obtained by Saslaw and Hamilton 24]. The shapes of the distribution function as a function of particle number $N$ and clustering parameter $b_{+}$are depicted in the figures 4, 5, 6, and 7,

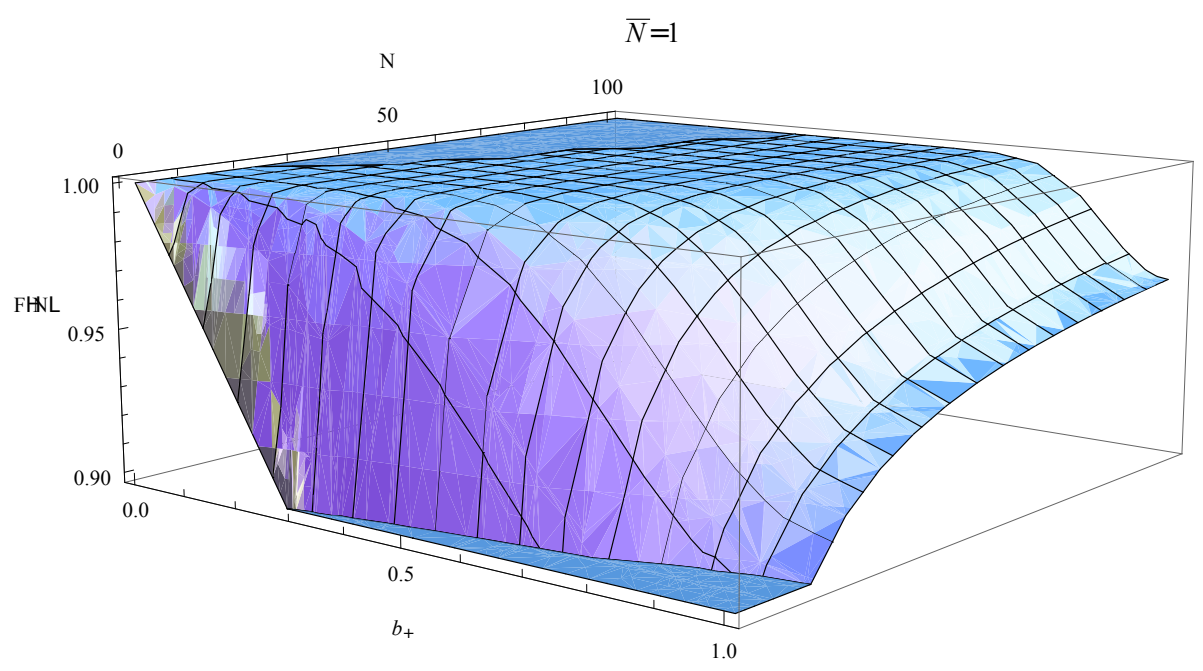

FIG. 4: For $\bar{N}=1$, the distribution function vs. clustering parameter $b_{+}$.

The behavior of the distribution function in two-dimensions is also shown in 8 , From the graph, it can be seen that the distribution function flattens as the value of the correction factor is increased. From the plot, we also deduce that the correction parameter shifts the peak downwards without changing the basic structure of the curve.

\section{POWER LAW FOR TWO-POINT CORRELATION FUNCTION}

Large scale surveys have shown that galaxies are not uniformly distributed in the universe [25]. Galaxies cluster on the scales of $\mathrm{Mpc}$ as well as in large-scale diverse structures on the scales of tens of Mpc. To describe clustering at a large scale, two types of statistics are commonly used. One is the two-point correlation function $(\xi(r))$, and the other is the power spectrum of object distribution, which is described as the correlation function's Fourier transform.

Here, we analyze the behavior of the correlation function in the presence of a corrected Hamiltonian. The assumption made by Peebles about the power-law form of the correlation 


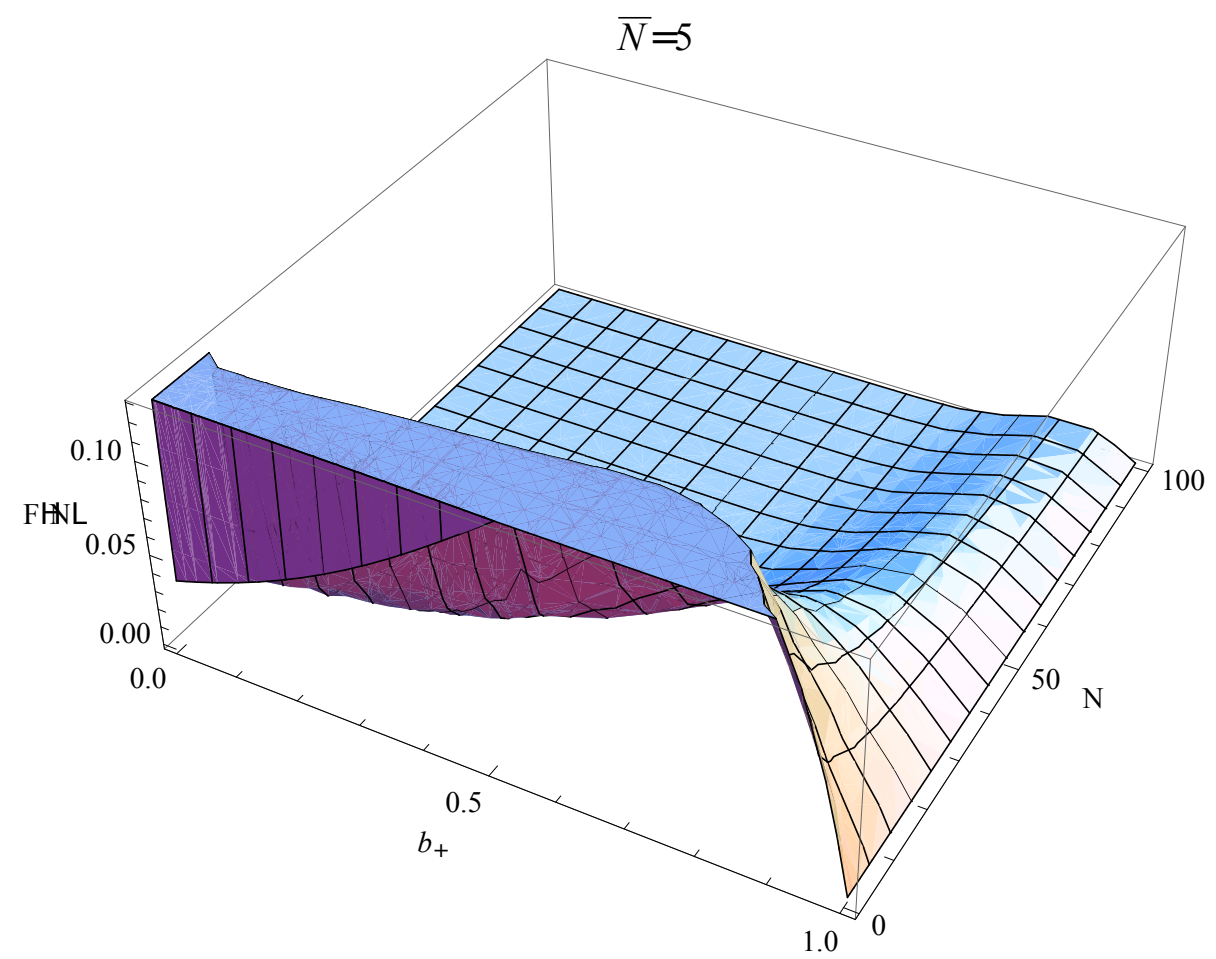

FIG. 5: For $\bar{N}=5$, the distribution function vs. clustering parameter $b_{+}$.

function, which describes the probability of finding another object within a given radius when the first object is identified [26], has been confirmed through $N$-body computer simulations [27].

The effect of modified potential on the power-law form of the correlation function can be visualized by writing the clustering parameter $b_{+}$in the following form [28]:

$$
b_{+}=\frac{\bar{n}}{6 T} \int\left[\frac{G M^{2}}{\left(r^{2}+\epsilon^{2}\right)^{1 / 2}}+B r^{2}\right] \xi_{2}(n, R, T) d V,
$$

where $\bar{n}$ is the particle number density.

When the above equation is differentiated with respect to $V$, it yields

$$
\begin{aligned}
\frac{\partial b_{+}}{\partial V} & =\frac{\bar{n}}{6 T} \frac{\partial}{\partial V} \int\left[\frac{G M^{2}}{\left(r^{2}+\epsilon^{2}\right)^{1 / 2}}+B r^{2}\right] \xi_{2}(n, R, T) d V \\
& +\frac{1}{6 T}\left(-\frac{\partial \bar{\rho}}{\partial V}\right) \int\left[\frac{1}{\left(r^{2}+\epsilon^{2}\right)^{1 / 2}}+B r^{2}\right] \xi_{2}(n, R, T) d V
\end{aligned}
$$

where we have used $\frac{\partial V}{\partial \bar{n}}=-\frac{\bar{n}}{V}$. 


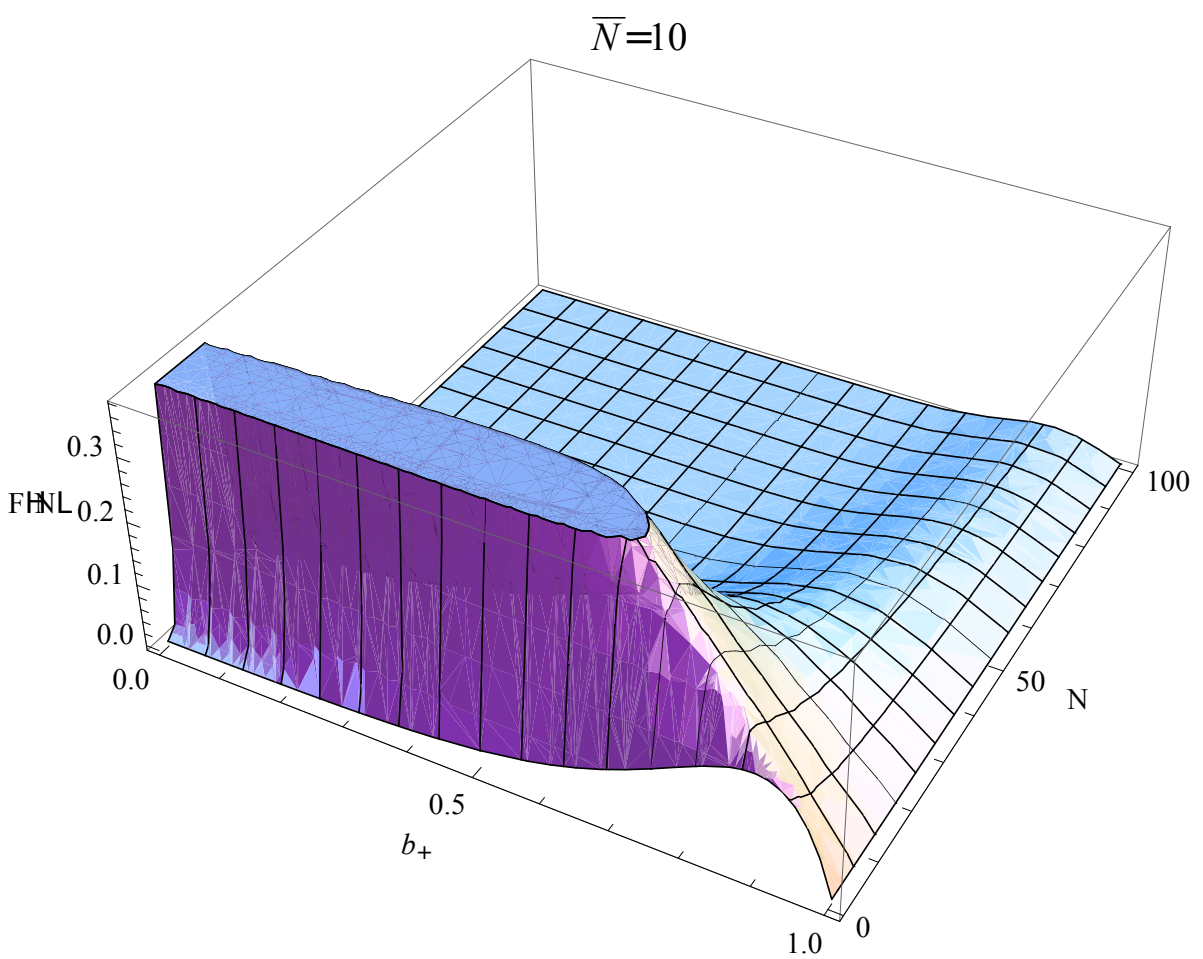

FIG. 6: For $\bar{N}=10$, the distribution function vs. clustering parameter $b_{+}$.

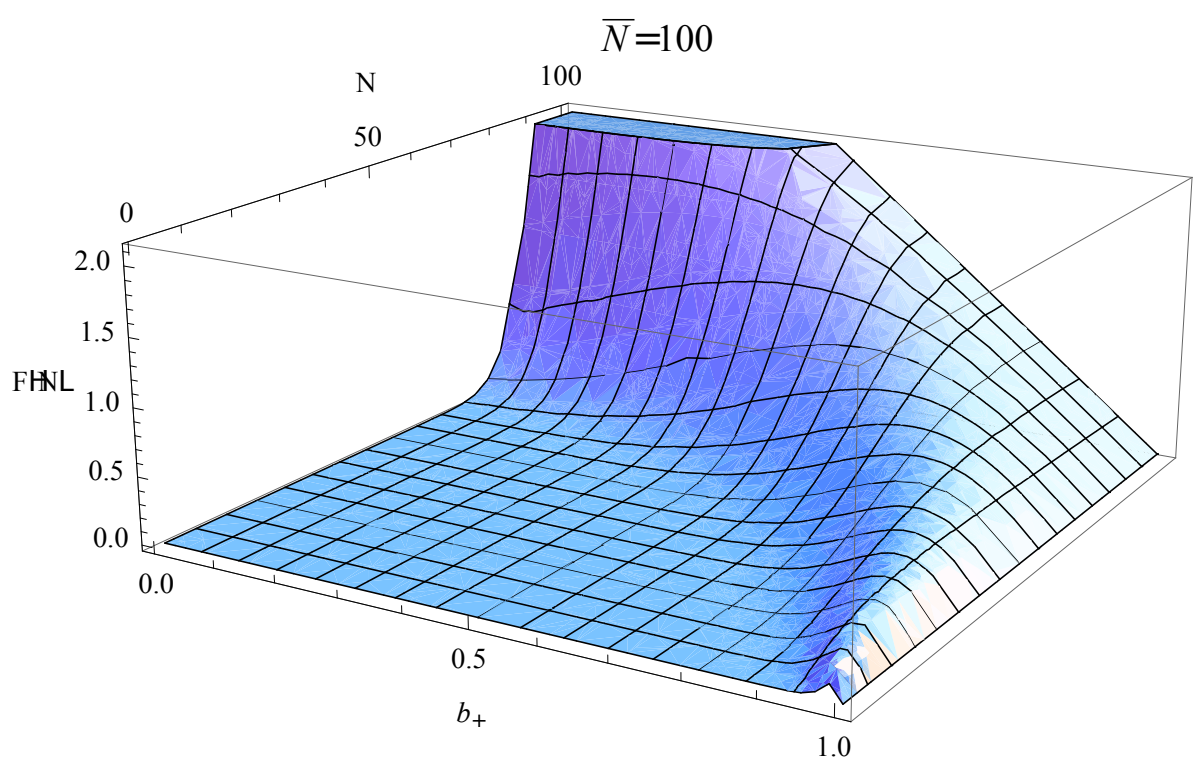

FIG. 7: For $\bar{N}=100$, the distribution function vs. clustering parameter $b_{+}$. 


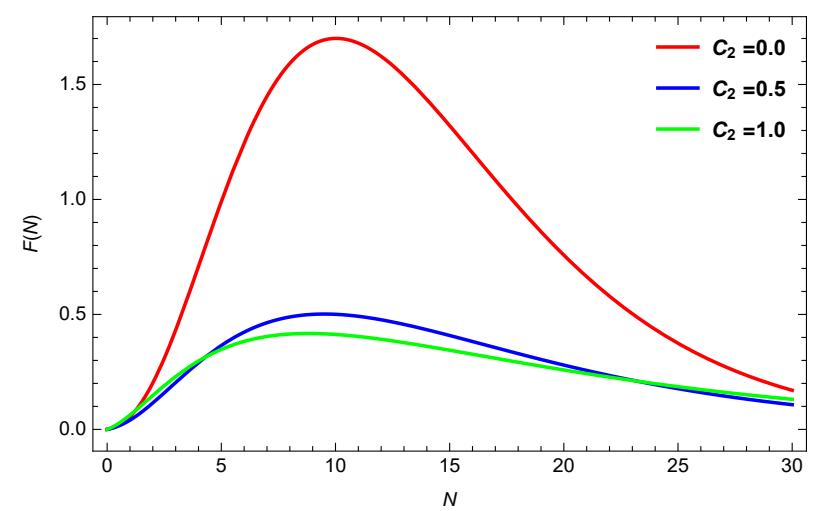

FIG. 8: The distribution function $F(N)$ 's behaviour as a function of particle number $N$. The red, blue, and green curves represent three different values for the correction parameter $C_{2}$. The red curve represents no correction, the blue curve represents $C_{2}=0.5$, and the green curve represents $C_{2}=1$.

Using the relation $\frac{\partial b_{+}}{\partial \bar{n}}=\frac{b_{+}\left(1-b_{+}\right)}{\bar{n}}$ in the preceding equation, we obtain the following relation for the power-law (for extended galaxies):

$$
\xi_{2}(r)=\frac{9 T b_{+}^{2}}{2 \pi \bar{n}}\left[\frac{G M^{2}}{\left(r^{2}+\epsilon^{2}\right)^{1 / 2}}+B r^{2}\right] .
$$

If we neglect the extended nature of galaxies and treat them as point particles, the above equation takes the form

$$
\xi_{2}(r)=\frac{9 T b_{+}^{2}}{2 \pi \bar{n}}\left[\frac{1}{r}+B r^{2}\right]
$$

\section{PHASE TRANSITION AND THE BEHAVIOR OF SPECIFIC HEAT}

The initial Poisson distribution (zero correlation) of a gravitationally driven many-body system evolves through different stages. The evolution can be described as the phase transition from uncorrelated phase to some positive value of correlation characterized by an order parameter $b_{+}$, i.e., $b_{+}=0$ to $b_{+}>0$. As clustering enhances via gravitational interaction, the homogeneity of the system is lost and lumps are created. The behavior of specific heat is an important indicator of a phase transition. We analyze the variation of the specific heat with the growth in the system temperature. 
The specific heat at constant volume is defined as

$$
C_{V}=\frac{1}{N}\left(\frac{\partial U}{\partial T}\right)_{V, N}
$$

Using equations (35) and (23), the expression for specific heat is

$$
C_{V}=\frac{3}{2}\left[\frac{1+6 C \eta-4 C^{2} \eta^{2}}{(1+C \eta)^{2}}\right]
$$

where $C=C_{1}+C_{2}$ has been substituted.

Initially, when there is no clustering i.e. $b_{+}=0$, the value of specific heat $C_{V}=3 / 2$. When the system becomes fully virialized i.e $b_{+}=1, C_{V}=-3 / 2$. In between these two extreme values there lies a critical value of temperature, $T=T_{C}$ at which $C_{V}$ takes the extremum (maximum) value

$$
\frac{\partial C_{V}}{\partial T}=0
$$

The value of the critical temperature for the system of galaxies interacting via the corrected Newtonian potential is given by

$$
T_{C}=\left[3 \frac{\bar{N}}{V}\left(G M^{2}\right)^{3}\left(C_{1}+C_{2}\right)\right]^{1 / 3} .
$$

In terms of critical temperature, the specific heat $C_{V}$ (36) can be expressed as follows:

$$
C_{V}=\frac{3}{2}\left[1-2 \frac{1-4\left(T / T_{C}\right)^{3}}{\left\{1+2\left(T / T_{C}\right)^{3}\right\}^{2}}\right] .
$$

Figure 9 shows that at $T=T_{C}, C_{V}=5 / 2$, which is a typical diatomic gas property. At this point, the system homogeneity breaks at the average inter-particle separation by the formation of bound binary systems. This marks the initiation of hierarchical phase transition at the lowest possible scale. The system symmetry breaking occurs on increasing scales, which is in principle different from the spontaneous phase transition commonly known.

\section{CONCLUSION}

A sphere of mass $M$ gravitates as a point particle of mass $M$ located at its center, according to Newton's "sphere-point" equivalence theorem, which connects terrestrial gravity to the orbital 


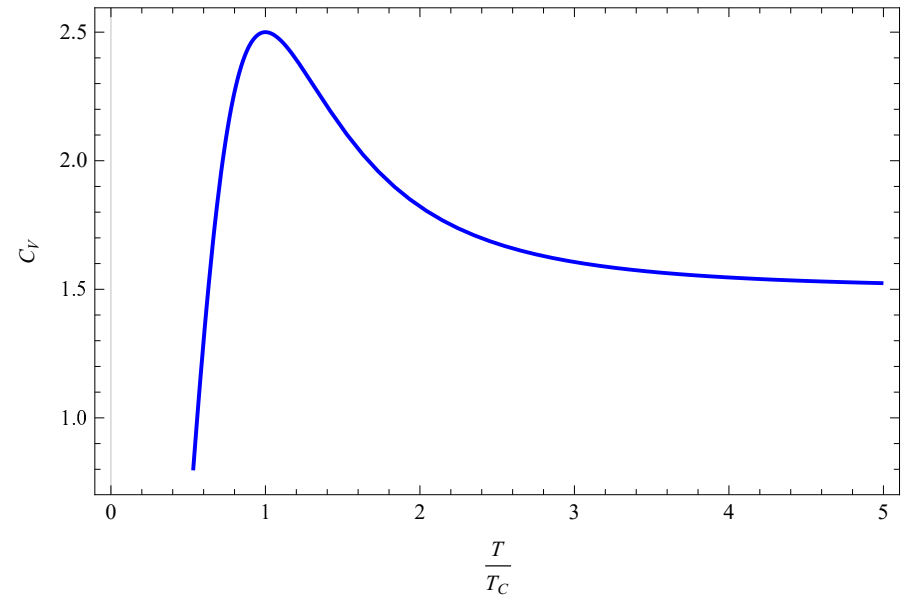

FIG. 9: The graph depicts the change in specific heat $C_{V}$ as a function of temperature $T / T_{C}$. It can be seen that the system symmetry breaks at $T=T_{C}$.

motion of planets in the solar system. The general solution that satisfies this theorem, $f(r)=$ $A r^{-2}+B r$, is the low energy limit of GR with a positive value of the cosmological constant. This connection is evident through the correspondence of the current observed value of the cosmological constant with the value of the cosmological term in the modified Newtonian gravity law. In the present paper, we have presented a quantitative study of the clustering of galaxies under this modified Newtonian gravity. It was observed that the corrected Newtonian potential modifies the clustering parameter, which reflects the effect of the correction on the clustering. We have calculated the partition function for the gravitationally interacting system of galaxies. We have used the grand partition function to calculate the various thermodynamic equations of state like Helmholtz free energy, entropy, pressure, and chemical potential. We also studied the effect of the modified potential on the spatial distribution of the galaxies. The correlation of the system particles is also studied under a modified Newtonian potential. The evolution of the system as characterised by the specific heat behaviour indicates the possibility of a phase transition around the critical temperature $T_{C}$.

[1] Hubble, E. P. (1925). Cepheids in spiral nebulae. Popular Astronomy, 33.

[2] Zwicky, F. (1933). Die rotverschiebung von extragalaktischen nebeln. Helvetica physica acta, 6, 
$110-127$.

[3] Kaiser, N. (1986). Evolution and clustering of rich clusters. Monthly Notices of the Royal Astronomical Society, 222(2), 323-345.

[4] Jain, B. and Khoury, J., Jain, B., and Khoury, J. (2010). Cosmological tests of gravity. Annals of Physics, 325(7), 1479-1516.

[5] Capozziello, S., Faizal, M., Hameeda, M., Pourhassan, B., Vincenzo, S., and Upadhyay, S., (2018). Clustering of galaxies with $\mathrm{f}(\mathrm{R})$ gravity. Monthly Notices of the Royal Astronomical Society, 474, $2430-2443$.

[6] Hameeda, M., Pourhassan, B., Faizal, M., Masroor, C. P., Ansar, R., and Suresh, P. K. (2019). Modified theory of gravity and clustering of multicomponent system of galaxies. The European Physical Journal C, 79, 769.

[7] Hameeda, M., Pourhassan, B., Rocca, M. C., and Faizal, M. (2021). Finite Tsallis gravitational partition function for a system of galaxies. General Relativity and Gravitation, 53, 41.

[8] Capozziello, S., Faizal, M., Hameeda, M., Pourhassan, B., and Salzano, V. (2021). Logarithmic corrections to Newtonian gravity and large scale structure. The European Physical Journal C, 81, 352.

[9] Hameeda, M., Pourhassan, B., Rocca, M. C., and Brzo, A. B. (2021). Gravitational partition function modified by superlight braneworld perturbative modes. Physical Review D, 103, 106019.

[10] Upadhyay, S. (2017). Thermodynamics and galactic clustering with a modified gravitational potential. Physical Review D, 95(4), 043008.

[11] Khanday, A. W., Upadhyay, S., and Ganai, P. A. (2021). Galactic clustering under power-law modified newtonian potential. General Relativity and Gravitation, 53(6), 1-19.

[12] Hameeda, M., Upadhyay, S., Faizal, M., and Ali, A. F. (2016). Effects of cosmological constant on clustering of Galaxies. Monthly Notices of the Royal Astronomical Society, 463(4), 3699-3704.

[13] Pourhassan, B., Upadhyay, S., Hameeda, M., and Faizal, M. (2017). Clustering of galaxies with dynamical dark energy. Monthly Notices of the Royal Astronomical Society, 468(3), 3166-3173.

[14] Hameeda, M., Pourhassan, B., Masood, S., Faizal, M., Wang, L. G., and Abass, S. (2020). The large scale structure formation in an expanding universe. arXiv preprint arXiv:2007.03442.

[15] Gurzadyan, V. G. (1985). The cosmological constant in the McCrea-Milne cosmological scheme. The Observatory, 105, 42-43.

[16] Gurzadyan, V. G., De Paolis, F., Nucita, A. A., Kashin, A. L., Amekhyan, A., Sargsyan, S., ... and Vetrugno, D. (2018). Messier 81's Planck view versus its halo mapping. Astronomy and Astrophysics, 609, A131.

[17] Kravtsov, A. V. (2013). The size-virial radius relation of galaxies. The Astrophysical Journal Letters, 
764(2), L31.

[18] Gurzadyan, V. G., and Stepanian, A. (2021). Hubble tension vs two flows. The European Physical Journal Plus, 136(2), 1-12.

[19] Ahmad, F., Saslaw, W. C., and Bhat, N. I. (2002). Statistical mechanics of the cosmological manybody problem. The Astrophysical Journal, 571(2), 576.

[20] Ahmad, F. (1987). Gravitational potential energy of a pair of overlapping galaxies. The Astrophysical Journal, 129, 1-9.

[21] Saslaw, W. C., and Hamilton, A. J. S. (1984). Thermodynamics and galaxy clustering-Nonlinear theory of high order correlations. The Astrophysical Journal, 276, 13-25.

[22] Saslaw, W. C., and Crane, P. (1991). The scale dependence of galaxy distribution functions. The Astrophysical Journal, 380, 315-319.

[23] Wen, Z. L., Han, J. L., and Liu, F. S. (2012). A catalog of 132,684 clusters of galaxies identified from Sloan Digital Sky Survey III. The Astrophysical Journal Supplement Series, 199(2), 34.

[24] Saslaw, W. C., and Hamilton, A. J. S. (1984). Thermodynamics and galaxy clustering-Nonlinear theory of high order correlations. The Astrophysical Journal, 276, 13-25.

[25] Hawkins, E., Maddox, S., Cole, S., Lahav, O., Madgwick, D. S., Norberg, P., ... and Taylor, K. (2003). The 2dF Galaxy Redshift Survey: correlation functions, peculiar velocities and the matter density of the Universe. Monthly Notices of the Royal Astronomical Society, 346(1), 78-96.

[26] Peebles, P. J. E. (2020). The large-scale structure of the universe (Vol. 98). Princeton university press.

[27] Itoh, M., Inagaki, S., and Saslaw, W. C. (1993). Gravitational clustering of galaxies: Comparison between thermodynamic theory and N-body simulations. IV-The effects of continuous mass spectra. The Astrophysical Journal, 403, 476-496.

[28] Iqbal, N., Ahmad, F., and Khan, M. S. (2006). Gravitational clustering of galaxies in an expanding universe. Journal of Astrophysics and Astronomy, 27(4), 373-379. 